\title{
Nuclear background affects frequency dynamics of mitochondrial DNA variants in Drosophila simulans
}

\author{
LOREDANA NIGRO \\ Dipartimento di Biologia, Università di Padova, Via Trieste 75, 35121 Padova, Italy
}

\begin{abstract}
Transplasmic lines, in which the original mitochondrial DNA (mtDNA) had been completely replaced by the mtDNA of the donor, were constructed to test for selection on mtDNA variants and to investigate the effects of nuclear-cytoplasmic interactions in Drosophila simulans. These lines were used to set up 12 experimental populations putting in competition two mtDNA variants with two different starting frequencies. Population cages were maintained for 20 generations and periodically sampled to monitor haplotype frequencies. Evidence is provided for a complete reversal of the forces acting on the frequency dynamics of the mtDNA variants according to the lines used to establish the population cages. In the cages where the competing lines had both the original nuclear and mtDNA, the sill type mtDNA variant seemed to be favoured but the situation was reversed when this variant was put in a different nuclear background.
\end{abstract}

Keywords: mt DNA, nuclear-cytoplasmic interactions, transplasmic lines.

\section{Introduction}

Mitochondrial DNA variants are often implicitly considered to be neutral markers and used as such in population studies but molecular analyses suggest that not all mtDNA mutations are neutral. A large part of the molecule seems to be under selective constraints. Substitutions seem in fact to occur more frequently in some regions than others (Simon, 1991) but it is still not clear whether they can affect individual fitness and whether these effects depend also on the involvement of nuclear genes.

Examples of mtDNA variants with phenotypic expression are known mainly in plants and yeast and in a few cases in mammalian cells and in maternally-transmitted human diseases (Beale \& Knowles, 1978; Dewey et al., 1986; King \& Attardi, 1988; Zeviani et al., 1989).

Experimental tests to detect selection on mtDNA have mostly used competition in population cages. The results were explained, in one case, by incompatibilitycausing microorganisms in the founding line, with no need to invoke selection on mtDNA (Nigro \& Prout, 1990). However, in other cases (MacRae \& Anderson, 1988; Fos et al., 1990) they seemed to be due to nuclear-cytoplasm interactions rather than to selection on the mtDNA variants per se.

At the biochemical level, nuclear and mitochondrial genes must be integrated in their expression (the mitochondrial protein ATPase, cytochrome oxidase and cytochrome $\mathrm{b}$ have subunits encoded also by nuclear genes). Thus any attempt to address the adaptive significance of mtDNA variants should consider the importance of the nuclear-cytoplasmic interactions.

In principle, if different selective pressures on mtDNA variants exist, they could result in geographical variation, different haplotypes being favoured and predominating in different areas.

Indeed, the distribution of $D$. simulans mtDNA variants has revealed a very puzzling situation (BabaAissa et al., 1988). Most individuals collected from all over the world showed the same restriction pattern (sill type). Two different cleavage morphs (sil and sillI types) were detected only in individuals from IndoPacific islands.

This situation has been recently confirmed by Satta and Takahata (1990) emphasizing the long persistence times of these distinct types of mtDNA in $D$. simulans. The three distinct ancestral lineages have been present in this species for over one million years, which seems 
too long for neutral lineages to persist in a randomly mating population. To inquire further into this anomalous situation, and keeping in mind also the importance of the nuclear-cytoplasmic interaction, a population cage experiment has been performed to measure the fitnesses of two different mtDNA variants of $D$. simulans.

\section{Materials and methods}

\section{Drosophila strains}

Two different strains were used in the experiment. One of the strains belonged to the sill type, the most common one, and was collected in 1985 in Padova (Italy) and the other one to the rare morph, sill type, and was collected in Nasr'allah (Tunisia, Africa) in 1983 (kindly provided by Catherine MontchampMoreau).

Both strains were tested to exclude any bacterial infection that could affect the compatibility of the crosses (Hoffmann et al., 1986) by making reciprocal crosses between the strains and counting the resulting progeny, as shown elsewhere (Nigro \& Prout, 1990).

It is known (Baba-Aissa et al., 1988) that the mtDNA of the two strains shows a different restriction pattern when digested with $\mathrm{HpaII}$ which allows an easy characterization of the two variants by subjecting the digests to electrophoresis on 0.8 per cent agarose gel and staining it with ethidium bromide.

The importance of the interaction between nuclear and mitochondrial genomes has been investigated using transplasmic lines. The lines were obtained by injecting, before pole cell formation, the cytoplasm from the eggs of silI type into the posterior end of eggs of sill type according to the technique described by Santamaria (1987), as reported elsewhere (Nigro, 1991).

As the sill type and silII type mtDNA restriction patterns differ after digestion with HpaII it is possible to test the success of cytoplasm transfer by looking for the heteroplasmy. Several different sublines were established from an injected heteroplasmic female. Some of them remained heteroplasmic whereas some reverted to the original mitochondrial state and some switched to the mitochondrial morph of the donor strain. In the last case the original nuclear-mitochondrial DNA association was disrupted (Fig. 1).

The transplasmic line used in the experiment $\left(\right.$ siII $\left._{\text {injected }}\right)$ had the sill type mtDNA of the donor line from Italy on the nuclear background of the acceptor silII type from Tunisia. Reciprocal crosses were made between the founding lines in order to randomize the mtDNA differences with any nuclear genetic differ- ences. The progeny lines from these crosses were then expanded to build the population cages.

\section{Population cages and experimental design}

Twelve population cages, each with 700 inseminated females, were established in four different ways, as summarized in Table 1, and kept at a constant temperature of $23^{\circ} \mathrm{C}$.

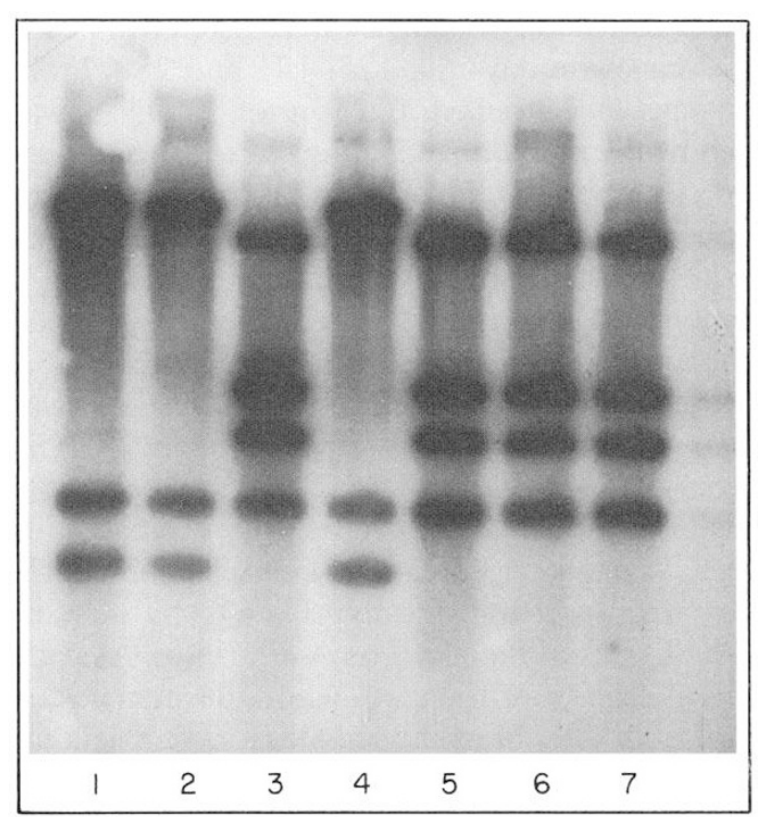

Fig. 1 Autoradiography of a Southern blot of mitochondrial DNA extracted from single females after the injection procedure and digested with HpaII. Lanes 1,2 and 4: pure sillI sublines. Lanes 3, 5-7: pure sill sublines.

Table 1 Twelve population cages were established with 700 inseminated females in each cage and with three replicates for each set of competition. Selective coefficients were evaluated by a model of linear frequency. $Y$ is a coefficient estimated for the linear change per generation and $s$ is an estimate of the selection coefficient back-transformed from $Y$ by $s=\sin ^{2}(Y / 2)$ where $Y$ is in radians

\begin{tabular}{lcccrr}
\hline $\begin{array}{c}\text { Population } \\
\text { cages }\end{array}$ & $\begin{array}{c}\text { Starting } \\
\text { frequency } \\
\text { sillI }\end{array}$ & $\begin{array}{c}\text { Linear } \\
\text { model } \\
\chi^{2}\end{array}$ & d.f. & $Y$ & \multicolumn{1}{c}{$s$} \\
\hline III vs. II inj & 0.7 & 0.60 & 1 & -0.0341 & -0.0003 \\
III vs. II inj & 0.3 & $10.30^{* *}$ & 1 & 0.1477 & 0.0054 \\
III vs. II & 0.7 & 0.48 & 1 & -0.0326 & -0.0003 \\
III vs. II & 0.3 & $5.06^{*}$ & 1 & -0.1064 & -0.0028 \\
\hline
\end{tabular}

$* P \leqslant 0.05,{ }^{* *} P \leqslant 0.01$. 
The cages were made of plexiglass (dimensions $30 \times 20 \times 15 \mathrm{~cm}$ ) with 12 openings for $4 \times 11 \mathrm{~cm}$ bottles with medium made of sucrose, agar, brewer's yeast and nipagin mold inhibitor. A continuous population was maintained by exchanging 6 bottles with fresh medium for the set of 6 older bottles every 20 days.

The initial frequency of the silII haplotype was 0.7 or 0.3 with three replicates for each set. In two sets of cages the sill type was put in competition with the silI $_{\text {injected }}$ type while in the other two sets it was in competition with the sill type.

Samples of around 40 females were taken from each of the 12 cages on days 50,125, 175 and 280. From each female an enriched fraction of mtDNA was extracted by the method of Coen et al. (1982) and then digested with HpaII. The digest was subjected to electrophoresis to monitor the frequency changes of the two mtDNA types over generations.

Tests on mtDNA frequency changes were made using the normal deviate, $Z$, to reject a null hypothesis of neutral drift. The intensity of selection was measured with a model of linear frequency, using arcsin transformations of mtDNA type frequencies, on the basis of the linear slope parameter $Y$, estimated for the linear change per generation. The selection coefficient, $s$, was back-transformed from $Y$ by $s=\sin ^{2}$ $(Y / 2)$ where $Y$ is in radians (Fos et al., 1990). As each population cage was founded with 700 females, a sample of $2000\left(N_{\mathrm{f}}\right)$ breeding females for each generation seemed sufficient for the analyses. The effective population size is usually considered to correspond roughly to the number of breeding individuals and for mtDNA, which is maternally transmitted, that corresponds to the number of breeding females.

\section{Results}

The frequency changes of the sill type in the 12 replicates for the four sets of cages are shown in Fig. 2. The directional trends observed in the two kinds of competition were completely different. In the cages where the competition was between strains having both of the original nuclear-cytoplasmic associations, the frequency of the silII type mtDNA decreased in all the replicates whereas it increased when it was in competition with the transplasmic strain having the sill mtDNA type in the silll type nuclear background.

As the total $G$ test $($ d.f. $=6)$ among the replicates measured for each of the four different sets of cages was never significant, the values were combined (Fig. 3 ). The sill haplotype placed with its own nuclear background tends to be positively selected but is negatively selected when put in the nuclear background of the other strain. The disruption of the nuclear-cyto-

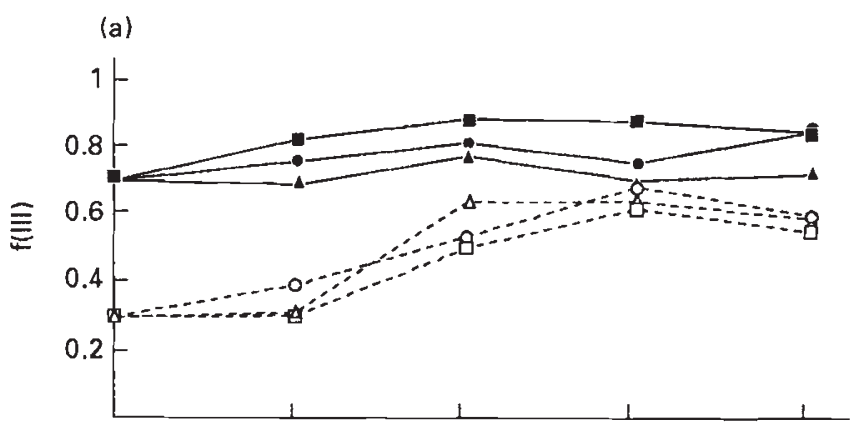

(b)

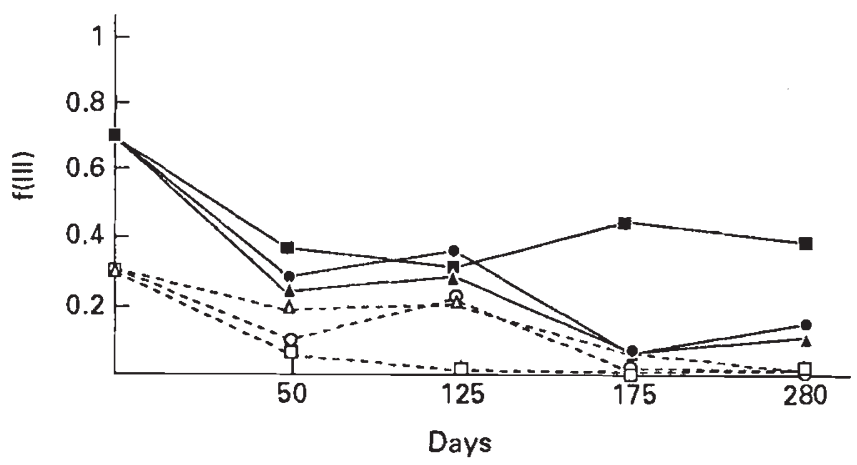

Fig. 2 Results on sampling populations: (a) frequency changes in the cages with silII type vs. sill ${ }_{\text {injected }}$ type mtDNA, (b) frequency changes in the cages with silll type vs. sill type mtDNA. $f($ III $)$ : relative frequency of the silll type mtDNA. Solid lines are replicates for populations started with a frequency of 0.70 . Dashed lines are replicates for populations started with a frequency of 0.30 .

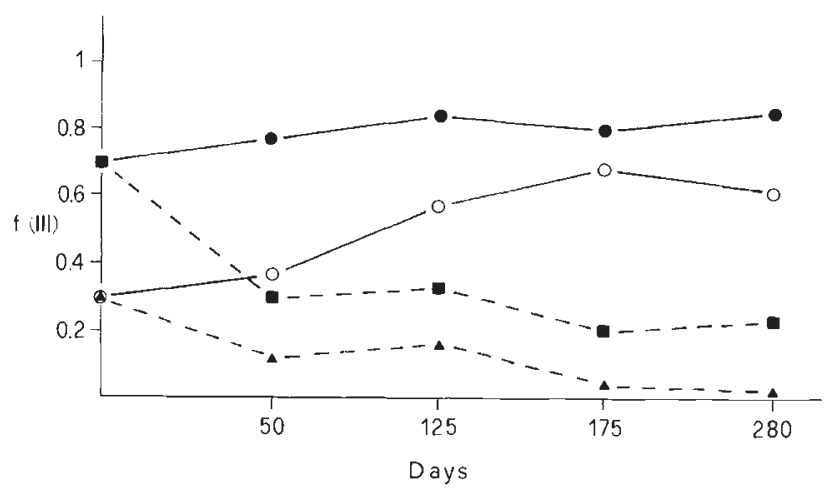

Fig. 3 Solid lines are means of three replicates in the cages

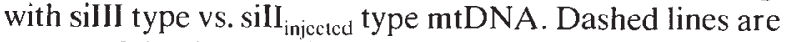
means of the three replicates of cages with sillI type vs. sill type mtDNA. $f($ III $)$ : relative frequency of the siIII type mtDNA.

plasmic association seems to determine a complete reversal of the trend for the sill frequency changes.

The $Z$ values, measuring mtDNA frequency changes, departed significantly from the null hypothesis of neutral drift for the four test generations, except 
in the cages with sillI vs. silI ${ }_{\text {injected }}$ from $F_{0}$ to the first sampling, corresponding roughly to the second generation $(Z=0.87$ and $Z=0.78$ for the cages with $f(\operatorname{silII})$ of 0.7 and 0.3 , respectively); however, the $Z$ values in this set of cages became significant between the first and the second samplings, $(Z=3.6, P=0.002$ and $Z=2.5$, $P=0.002$ in the cages with $f($ sillI $)$ of 0.7 and 0.3 ) showing just a slower increase of the sillI type.

The direction and the rapidity of frequency changes do not depend only on the mtDNA type and can be completely reversed when the original cyto-nuclear association is destroyed.

The selection coefficients $(s)$ acting on the different nuclear-cytoplasmic associations, evaluated by a model of linear frequency change using gene frequency arcsin transformation, are shown in Table 1. In the cages with $f($ silII $)$ of 0.3 the $s$ values are larger than $1 / N_{\mathrm{f}}$, where $N_{\mathrm{f}}$ is taken as 2000 , the number of breeding females that for mtDNA can be put equal to the effective population size for organelle genes (Fos et al., 1990). According to Hartl and Clark (1989) s values larger than $1 / N_{\mathrm{f}}$ can suggest the existence of some sort of selection to explain the frequency changes observed. In the cages with $f($ sillI $)$ of 0.7 there is no evidence of selection.

\section{Discussion}

The issue of the selective effects of the mtDNA polymorphism has been addressed both at the theoretical and the experimental level (Gregorious \& Ross, 1984; Clark, 1984, 1985; Clark \& Lyckegaard, 1988). It is known that the rate of nucleotide substitutions can be very different in different regions of the mtDNA but the possible adaptive significance of the organelle DNA variation in higher eukaryotes is still under debate.

In their study Clark and Lyckegaard (1988) analysed the influence of cytoplasmic variation on viability in $D$. melanogaster. They observed a significant nuclear-cytoplasmic interaction on second chromosome segregation in the experiment using flies of diverse geographical origin but it was not possible to ascribe the cytoplasmic effect with certainty to mtDNA.

MacRae and Anderson (1988) used population cages and put in competition two mtDNA variants of $D$. pseudoobscura. They suspected some sort of sporadic selection for mtDNA could be taking place but it was the 'last resort' explanation. They also suggested that both nuclear and mitochondrial genomes could be involved in the mtDNA frequency changes observed in the population cages.

A similar experiment carried out by Fos et al. (1990) once again showed the importance of the nuclear back- ground with which the mtDNA is associated in explaining the frequency changes observed.

While in all these cases there was no clear evidence of selection acting on mtDNA differences, the importance of the cyto-nuclear association was always evident.

The involvement of different factors in the cross-talk between nuclear and mitochondrial genomes has been recently shown in the case of human mitochondrial myopathy where a nuclear-coded protein can determine deletion of the mitochondrial genome in a heritable way (Zeviani et al., 1989).

Apart from Drosophila (De Stordeur et al., 1989; Niki et al., 1989), replacement of the resident mtDNA by an exogenous one has been obtained only in human cells (King \& Attardi, 1989) where the transformation appeared to have effects on fitness. The transformants were in fact drug-resistant whereas the donor cells were very sensitive. It was also shown that differences in reciprocal compatibility of the nuclear and mitochondrial genomes played a crucial role in determining the growth rate of different classes of transformants.

The results shown here suggest both nuclear-cytoplasmic interaction, with nuclear effects acting in different directions in each cytoplasm, and selection on mtDNA.

The frequencies of the sill type mtDNA changed in opposite directions depending on the nuclear background with which it was associated. It was positively selected only when kept with its own nuclear background, suggesting that the type II nuclear genes were outcompeting the type III nuclear genes, in agreement with previous observations (Solignac et al., 1984, 1987).

Selection pressure on the sillI mtDNA type was shown to be present also in artificial heteroplasmons, where the silI type mtDNA type was seen to have an advantage over the silII mtDNA type of 57.7 per cent, and can also explain the significant difference in the efficiency of the injection of the sill type into the sillI type compared with the reciprocal injection (De Stordeur et al., 1989). The same kind of pressure can explain the anomalous spatial geographical distribution for these two mtDNA variants in $D$. simulans. The sill type is the only one found all over the world, including the distribution area of the silll type.

The selective parameters estimated on the basis of the linear model suggest selection effects on the frequency variation of the two variants. As the number of organelles can be represented by the number of breeding females $\left(N_{\mathrm{f}}\right)$, and knowing that any selective value bigger than $1 / N_{\mathrm{f}}$ could suggest the action of selection (Hartl \& Clark, 1989; Fos et al., 1990), it is possible to ascribe the frequency changes observed in the cages with $f($ siIII) of 0.3 to some sort of selection. 
The asymmetry of the selection operating in one case in favour of the sill type and in the other case against it stresses the risk of confusing selection acting on mtDNA variants alone, with effects also due to nuclear-cytoplasmic interaction. Most of the mitochondrially-coded proteins are subunits of enzymes for which the other subunits are encoded in the nucleus; the associations between subunits can determine strong cyto-nuclear interactions.

The construction of transplasmic lines, in which the original mitochondrial DNA is completely replaced by the mtDNA of the donor, can be a useful tool to clarify the two-way communication between the nucleus and the cytoplasm and to give additional evidence for the gene-cytoplasm interactions that could affect fitness components. Further experiments with different and controlled combinations of nuclear and mitochondrial DNA could provide more information about the level of compatibility between nuclear and mitochondrial genomes and also give insight into the functional effects of nuclear coded factors on mtDNA.

\section{References}

BABA-AISSA, F., SOLIGNAC, M., DENNEBOUY, N. AND DAVID, J. R. 1988. Mitochondrial DNA variability in Drosophila simulans: quasi absence of polymorphism within each of the three cytoplasmic races. Heredity, 61, 419-426.

BEALE, G. AND KNOWLES, J. 1978. Extranuclear Genetics. Edward Arnold, London.

CLARK, A. G. 1984. Natural selection with nuclear and cytoplasmic transmission. I. A deterministic model. Genetics, 107, 679-701.

CLARK, A. G. 1985. Natural selection with nuclear and cytoplasmic transmission. II. Test with Drosophila from diverse populations. Genetics, 111,97-112.

CLARK, A. G. AND LYCKEGAARD, E. M. S. 1988. Natural selection with nuclear and cytoplasmic transmission. III. Joint analysis of segregation and mtDNA in Drosophila melanogaster. Genetics, 118, 471-481.

COEN, E. S., THODAY, J. N. AND DOVER, G. 1982. Rate turnover of structural variants in the rDNA gene family of Drosophila melanogaster. Nature, 295, 564-568.

DE STORDEUR, E., SOLIGNAC, M., MONNEROT, M. AND MONOULOU, J. C. 1989. The generation of transplasmic Drosophila simulans by cytoplasmic injection: effects of segregation and selection on the perpetuation of mitochondrial DNA heteroplasmy. Mol. Gen. Genet., 220, 127-132.

DEWEY, R. E., LEVINGS, C. S., III, AND TIMOTHY, D. H. 1986. Novel recombinations in the maize mitochondrial genome produce a unique transcriptional unit in the Texas malesterile cytoplasm. Cell, 44, 439-449.

FOS, M., DOMinqueZ, M. A., LATORRE, A. AND MOYA, A. 1990. Mitochondrial DNA evolution in experimental populations of Drosophila subobscura. Proc. Natl. Acad. Sci. U.S.A., 87, 4198-4201.

GREgorious, H. R. AND ROSS, M. D. 1984. Selection with gene-cytoplasm interactions. I. Maintenance of cytoplasmic polymorphism. Genetics, 107, 165-178.

HaRTL, D. AND Clark, A. 1989. Principles of Population Genetics. Sinauer, Sunderland, MA.

HOFFMANN, A. A., TURELLI, M. AND SIMMONS, G. M. 1986. Unidirectional incompatibility between populations of Drosophila simulans. Evolution, 40, 692-701.

KING, M. P. AND ATTARDI, G. 1988. Injection of mitochondria into human cells leads to rapid replacement of the endogenous mitochondrial DNA. Cell, 52, 811-919.

MACRAE, A. F. AND ANDERSON, w. w. 1988. Evidence of neutrality of mitochondrial DNA haplotypes in Drosophila pseudobscura. Genetics, 120, 485-494.

NIGRO, L. 1991. The effect of heteroplasmy on cytoplasmic incompatibility in transplasmic lines of Drosophila simulans showing a complete replacement of the mitochondrial DNA. Heredity, 66, 41-45.

NIGRO, L. AND PROUT, $\Upsilon$. 1990. Is there selection on RFLP differences in mitochondrial DNA? Genetics, 125, $551-555$

NIKI, Y., ChIGUSA, S. J. AND matsuURA, E. T. 1989. Complete replacement of mitochondrial DNA in Drosophila. Nature, 341, 551-552.

SANTAMAR1A, P. 1987. Injecting eggs. In: Roberts, D. B. (ed.) Drosophila: a Practical Approach, pp. 159-173. IRL Press, Oxford.

SATTA, Y. AND Takahata, N. 1990. Evolution of Drosophila mitochondrial DNA and the history of the melanogaster subgroup. Proc. Natl. Acad. Sci. U.S.A., 87, 9558-9662.

SIMON, C. 1991. Molecular systematics at the species boundary: exploiting conserved and variable regions of the mitochondrial genome of animals via direct sequencing from amplified DNA. In: Hewitt, G. M., Johnston, A. W. B. and Young, J. P. W. (eds) Molecular Techniques in Taxonomy, pp. 33-71. Springer-Verlag, Berlin.

SOLIGNAC, M., GENERMONT, J., MONNEROT, M., MONOULOU, J. C 1984. Genetics of mitochondria in Drosophila: mtDNA inheritance in heteroplasmic strains of D. mauritiana. Mol. Gen. Genet., 197, 183-188.

SOLIGNAC, M., GENERMONT, J., MONNEROT, M., MONOULOU, J. C. 1987. Drosophila mitochondrial genetics: evolution of heteroplasmy through germ line division. Genetics, 117 , 687-696.

ZEVIANI, M., SERVIDEI, S., GELLERA, C., BERTIN1, E., DI MAURO, S. AND DI DONATO, S. 1989. An autosomal dominant disorder with multiple deletions of mitochondrial DNA starting at the D-loop region. Nature, 339, 309-311. 